\section{Case Reports in Ophthalmology}

\title{
Is Spectral-Domain Optical Coherence Tomography Always Able to Detect the Anti-Vascular Endothelial Growth Factor Action on Neovascular Membrane?
}

\author{
Luigi Borgia $^{a}$ Chiara Del Noce $^{b}$ Michele Iester $^{b}$ \\ ${ }^{a}$ Casa di Cura Clinica Montallegro, Genoa, Italy; ${ }^{b}$ Laboratorio clinico anatomo-funzionale \\ per la diagnosi e il trattamento del glaucoma e della malattie neurooftalmologiche, \\ Clinica Oculistica, DiNOGMI, University of Genoa, Genoa, Italy
}

\section{Keywords}

Spectral-domain optical coherence tomography - Age-related macular degeneration . Intravitreal injection

\begin{abstract}
Purpose: To show the presence of an active neovascular membrane in age-related macular degeneration even if optical coherence tomography (OCT) does not detect intra- or subretinal edema. Methods: This is a retrospective case report. During the follow-up after the intravitreal injection, 3 patients showed no intraretinal or subretinal edema by OCT; however, there was a progressive reduction in their visual acuity; thus, a fluorangiography (FA) examination was performed. Results: In these 3 cases, FA showed an active neovascular network. Conclusion: OCT could show a real reduction in the edema, but it is not always able to detect neovessel presence. Intravitreal injection could improve the vessel permeability without care and delete the neovascular network.




\section{Introduction}

Age-related macular degeneration (ARMD) is a late-onset, multifactorial neurodegenerative disease [1]. Focal detachment of the retinal pigment epithelium (RPE), new blood vessel growth between Bruch's membrane and the retina, and outer retinal atrophy are the lesions that can cause loss of central vision in advanced ARMD, which can either involve choroidal neovascularization or be non-neovascular. Patients with geographic atrophy ARMD may display a broad spectrum of clinical characteristics based upon drusen size and ARMD pigmentary abnormalities, such as hypopigmentation and hyperpigmentation [2].

Vascular endothelial growth factor (VEGF) is an important growth factor for angiogenesis and has been shown to be necessary in normal vascular development. VEGF is highly selective for vascular endothelial cells and induces angiogenesis by serving as a potent endothelial cell mitogen [3]. It has been shown to be secreted by hypoxic RPE cells and induces endothelial cell proliferation and retinal vascular permeability. VEGF has been identified as a major mediator of retinal ischemia-associated neovascularization. VEGF overexpression induces endothelial cell proliferation and increases vascular permeability, properties that can be detected clinically as the presence of subretinal fluid or enlarging choroidal neovascularization. Anti-VEGF therapy might reduce subretinal fluid, a theoretical possibility with VEGF inhibition, resulting in short-term vision improvement [4].

In this case report, we showed the presence of an active neovascular membrane in ARMD even if optical coherence tomography (OCT) does not detect intra- or subretinal edema.

\section{Case Reports}

All the patients were diagnosed as having wet ARMD by L.B., and they were injected with anti-VEGF by M.I. In the 3 cases, ranibizumab was always used. Each patient had at least one intravitreal injection per month for at least 3 months [5] followed by an OCT evaluation 1 month after the last injection.

Patients were imaged using the Heidelberg Spectralis [Heidelberg retina angiography + OCT (Heidelberg Engineering, Heidelberg, Germany)] in the spectral-domain OCT mode, with a scan field of $30^{\circ}$ horizontally and $15^{\circ}$ vertically and 19-25 OCT horizontal sections (one section at least every $240 \mu \mathrm{m}$; Heidelberg Engineering, Spectralis hardware operating instructions; technical specifications, 2007, pp. 22-25).

In these patients, although no macular intraretinal fluid was present, there was a progressive reduction in their visual acuity; thus, fluorangiography (FA) was performed, and an active neovascular network was found in these 3 patients (fig. 1-3).

\section{Discussion}

There have been several well-publicized prospective, randomized studies that have demonstrated the efficacy of intravitreal injection of anti-VEGF drugs (ranibizumab, pegaptanib, and aflibercept; VEGF Trap-Eye) for patients with ARMD [6].

In our cases, we found no intraretinal edema by OCT with a progressive visual acuity loss. For this reason, FA was performed and a neovascular network was found even if the edema was resolved. We think that a predominant fibrotic neovascular network caused an 


\section{Case Reports in Ophthalmology}

alteration of the outer retinal layers with a worsening of the visual acuity also if intraretinal fluid was not present.

In most of the patients, anti-VEGF intravitreal injection showed an improvement of the patients' symptoms; however, intravitreal injections are not always able to resolve the new vessel angiogenic proliferation, but are able just to improve their impermeability. In this way, we can have a reduction in the retinal thickness due to the lack of intraretinal fluid, but with the visual acuity still worsening. Spectral-domain OCT was not able to detect the presence of an active neovascular network if the edema was not present [6].

In the literature, there are some studies that showed lack of activity. An anti-VEGF nonresponder is described as a patient who has loss or no change in either visual acuity and/or reading ability despite continuing monthly anti-VEGF therapy with persistent macular blood, intraretinal or subretinal fluid on OCT scans and leakage in FA. In a study on anti-VEGF unresponsiveness, Lux et al. [7] reported that the $45 \%$ of the patients were nonresponders to bevacizumab. In their study, it was also reported that the possibility of unresponsiveness to bevacizumab might be dependent on the initial lesion size, but was independent of the amount of intraretinal and subretinal fluid.

In two recent studies by Ladewig et al. [8] and Rich et al. [9], the proportion of nonresponders to bevacizumab was approximately 50\%. In this study, the proportion of nonresponders to all of anti-VEGF drugs was $32 \%$. Proportions of responsiveness to pegaptanib, bevacizumab and ranibizumab were 32,17 and 51\%, respectively. This was consistent with recent studies [10].

In the study by Turgut et al. [11], baseline OCT scans of nonresponder patients revealed some pathologies including foveal scarring, serous pigment epithelium detachment (PED), fibrovascular PED, hemorrhagic PED and vitreomacular traction syndrome. Thus, these may play a role in the unresponsiveness to anti-VEGF agents. Unresponsiveness may result from a number of factors including the development of subfoveal fibrosis or atrophy of the RPE and photoreceptors. In recent studies, it has been emphasized that the efficiency of antiVEGF agents might be reduced because of a large initial choroidal neovascular membrane having a greater fibrosis or lesser viable RPE or underlying genetic differences or an antiVEGF resistance [12].

In all these trials, patients were followed by using OCT and visual acuity based on the hypothesis that these drugs could block the angiogenesis and reduce the intraretinal edema. The importance of VEGF as a therapeutic target derives from its roles in two of the most basic processes within a typical lesion of advanced ARMD: neovascularization and vascular leakage.

OCT could show a real improvement of the edema, but it is not able to detect neovessel activity. The lack of FA in the trials could have hidden the loss of activity of these drugs on the angiogenesis, measuring only the effect on the permeability. In our cases, we found a very good improvement of retinal edema in patients, but when FA was performed, an active neovascular network was found (fig. 1-3).

The anti-VEGF capacity to improve vision in many patients has been shown in many studies, but unfortunately most of them are based on OCT scans and visual acuity and it is not possible to know whether the treatment was pointed out due to the neovessel growth or on the capacity to temporarily improve the neovessel permeability.

In the future, angio-OCT, which is not an invasive technique [13-15], could help clinicians to understand the macular neovascular network follow-up [16] and to detect the real activity of anti-VEGF in each patient and to decide upon the duration of the treatment. When an angio-OCT scan shows a neovessel network with a stable visual acuity and no intraretinal 
edema, the question will be whether to wait for a vision reduction and/or an intraretinal edema, or to start the retreatment anyway.

In conclusion, OCT could show a real reduction in the edema, but it is not always able to detect neovessel presence. Intravitreal injection could improve the vessel permeability without care and delete the neovascular network.

\section{Statement of Ethics}

The authors have no ethical conflicts to disclose.

\section{Disclosure Statement}

None of the authors has proprietary interests in the development and marketing of any of the products mentioned in the article. This research received no specific grant from any funding agency in public, commercial or not-for-profit sectors.

\section{References}

1 Ratnapriyaa R, Chewb EY: Age-related macular degeneration - clinical review and genetics update. Clin Genet 2013;84:160-166.

2 Birch DG, Liang FQ: Age-related macular degeneration: a target for nanotechnology derived medicines. Int J Nanomedicine 2007;2:65-77.

-3 Niu G, Chen X: Vascular endothelial growth factor as an anti-angiogenic target for cancer therapy. Curr Drug Targets 2010;11:1000-1017.

4 Kim R: Introduction, mechanism of action and rationale for anti-vascular endothelial growth factor drugs in age-related macular degeneration. Indian J Ophthalmol 2007;55:413-415.

-5 Spaide RF: Ranibizumab according to need: a treatment for age-related macular degeneration. Am J Ophthalmol 2007;143:679-680.

-6 Kimoto K, Kubota T: Anti-VEGF agents for ocular angiogenesis and vascular permeability. J Ophthalmol 2012;2012:852183.

7 Lux A, Llacer H, Heussen FMA, Joussen AM: Non-responders to bevacizumab (Avastin) therapy of choroidal neovascular lesions. Br J Ophthalmol 2007;91:1318-1322.

-8 Ladewig MS, Ziemssen F, Jaissle G, et al: Intravitreal bevacizumab for neovascular age-related macular degeneration (in German). Ophthalmologe 2006;103:463-470.

-9 Rich RM, Rosenfeld PJ, Puliafito CA, et al: Short-term safety and efficacy of intravitreal bevacizumab (Avastin) for neovascular age-related macular degeneration. Retina 2006;26:495-511.

10 Avery RL, Pieramici DJ, Rabena MD, Castellarin AA, Nasir MA, et al: Intravitreal bevacizumab (Avastin) for neovascular age-related macular degeneration. Ophthalmology 2006;113:363-372.

11 Turgut B, Tanyildizi R: The frequency of the nonresponsiveness to intravitreal injection of the antivascular endothelial growth factor agent in neovascular age related macular degeneration. Int J Ophthalmic Pathol 2013;2:3, DOI: 10.4172/2324-8599.1000119.

12 Wang Y, Wang VM, Chan C-C: The role of anti-inflammatory agents in age-related macular degeneration (AMD) treatment. Eye (Lond) 2011;25:127-139.

$\$ 13$ Tan 0, et al: En face Doppler total retinal blood flow measurement with $70 \mathrm{kHz}$ spectral optical coherence tomography. J Biomed Opt 2015;20:066004.

14 Wang Y, et al: In vivo total retinal blood flow measurement by Fourier domain Doppler optical coherence tomography. J Biomed Opt 2007;12:041215.

-15 Werkmeister RM, et al: Bidirectional Doppler Fourier-domain optical coherence tomography for measurement of absolute flow velocities in human retinal vessels. Opt Lett 2008;33:2967-2969.

16 Kuehlewein L, Bansal M, Lenis TL, Iafe NA, Sadda SR, Bonini Filho MA, De Carlo TE, Waheed NK, Duker JS, Sarraf D: Optical coherence tomography angiography of type 1 neovascularization in age-related macular degeneration. Am J Ophthalmol 2015;160:739-748. 


\section{Case Reports in Ophthalmology}
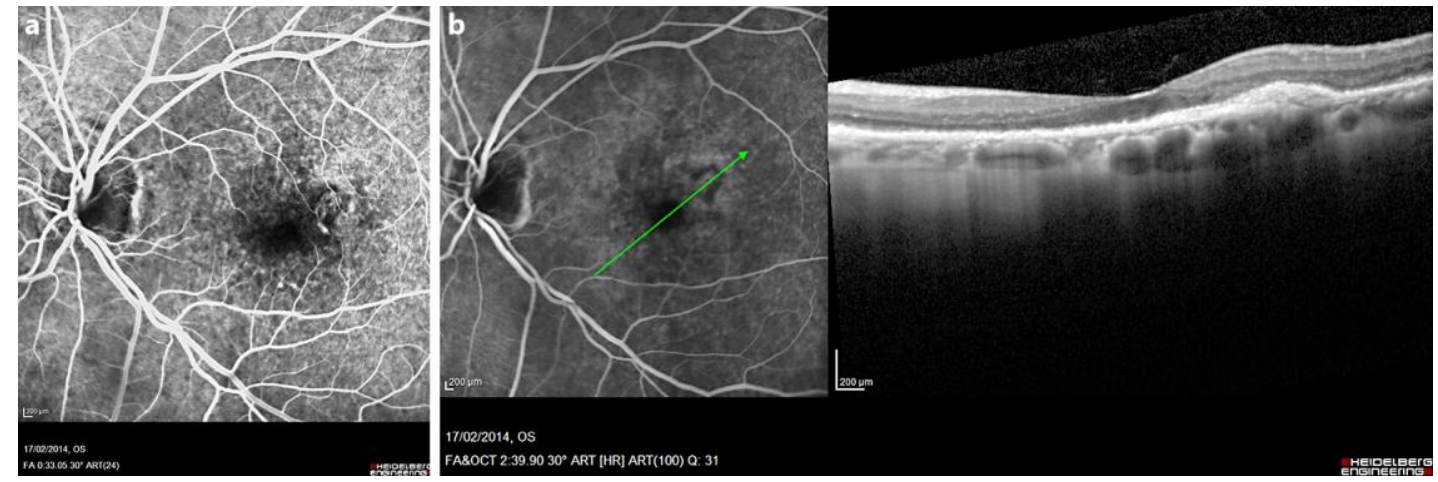

Fig. 1. OCT and FA: by using FA (a). An active membrane was present after 3 intravitreal injections, while no intraretinal edema was found in the OCT scans (b). Visual acuity was $6 / 10$ before intravitreal injection and $5 / 10$ after treatment.
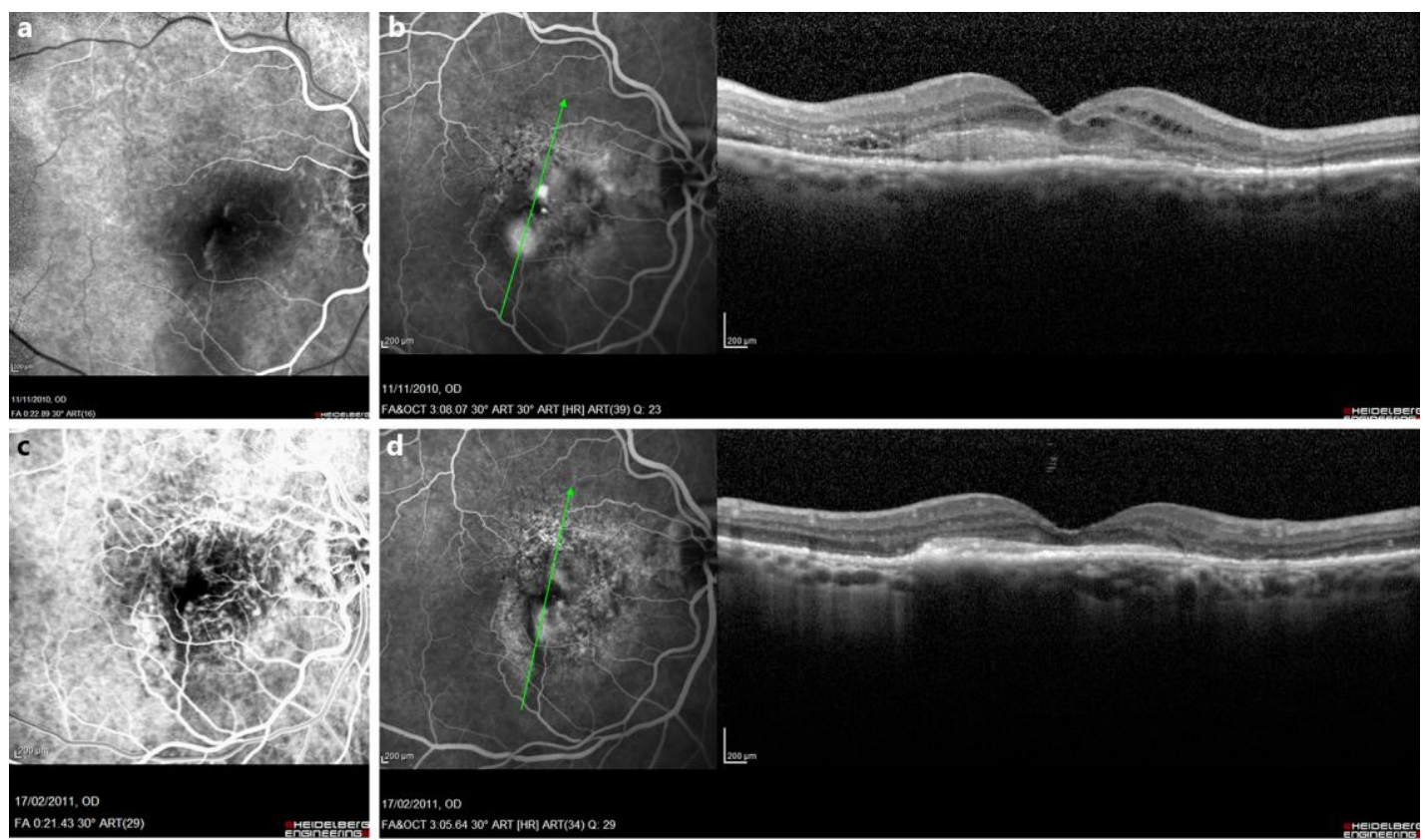

Fig. 2. a, b OCT and FA before intravitreal injections. c, d FA and OCT after 3 intravitreal injections. No intraretinal edema was found by OCT, but by FA an active membrane was still visible after 3 intravitreal injections. Visual acuity was 2/10 before intravitreal injections and 2/10 after treatment. 


\section{Case Reports in Ophthalmology}
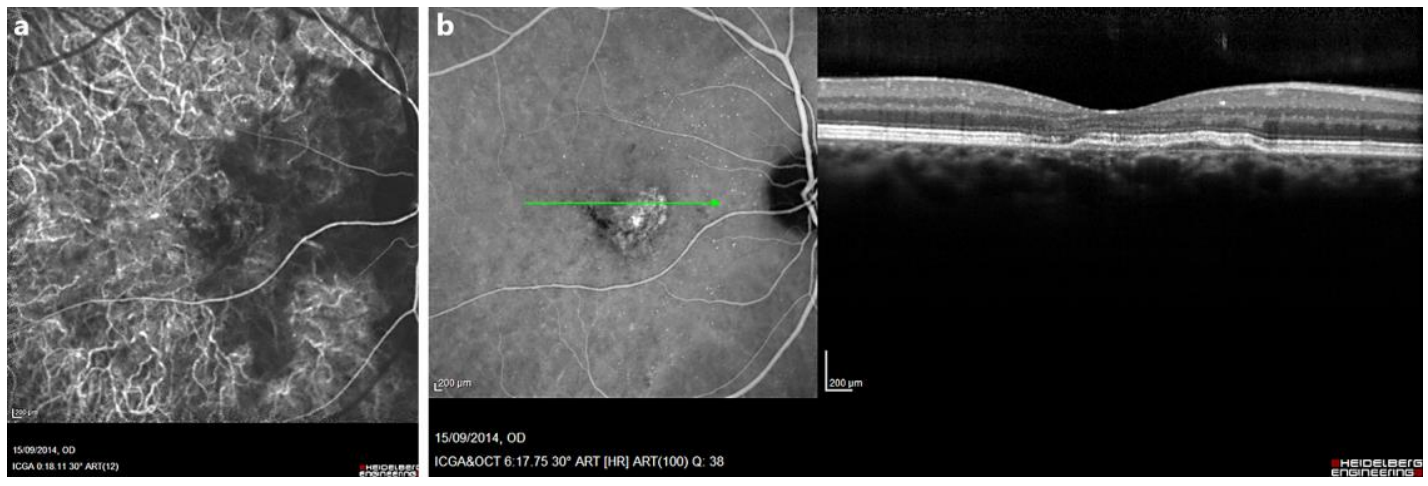

Fig. 3. OCT and indocyanine angiography (ICG): by using ICG (a), an active membrane was present and no intraretinal edema was found in the OCT scans (b). Visual acuity was 8/10 before intravitreal injections and $7 / 10$ after treatment. 